\title{
Towards a turbulence characterization in tidal energy sites. First results of THYMOTE project.
}

\author{
Sylvain Guillou, ${ }^{1, *}$, Jean-François Filipot ${ }^{2}$, Jérôme Thiébot $^{1}$, Grégory Germain $^{3}$, Nicolas \\ Chaplain $^{4}$, Maria Ikheunnicheu ${ }^{3}$, Rui Duart ${ }^{2}$, Benôt Gaurier $^{3}$, Adrien Bourgoin ${ }^{1}$, Philippe \\ Mercier $^{1}$, Riadh $\mathrm{Ata}^{5}$, Jérôme Laverne ${ }^{5}$, Sofiane Benhamadouche ${ }^{5}$, Aline Pieterse ${ }^{3}$, \\ Christophe Maisondieu ${ }^{3}$, Emmanuel Poizot ${ }^{1}$, Jean-Charles Poirier ${ }^{6}$, Cédric Auvray ${ }^{7}$, Elois \\ Droniou $^{8}$, Valentin Arramounet ${ }^{9}$, Grégory Pinon ${ }^{10}$
}

\author{
${ }^{1}$ Laboratoire Universitaire des Sciences Appliquées de Cherbourg, UNICAEN, 60 rue Max-Pol \\ Fouchet 50130 Cherbourg-en-Cotentin France \\ ${ }^{2}$ France Energies Marines, 15 rue Johannes Kepler, 29200 Brest \\ ${ }^{3}$ IFREMER, 29280 Plouzané, France \\ ${ }^{4}$ IXSurvey, 46 Quai François Mitterrand, 13600 La Ciotat \\ ${ }^{5}$ EDF-LNHE, 6 quai Watier, 78401 Chatou, cedex \\ ${ }^{6}$ SIRENHA, Technocampus Océan, 5 rue de l'Halbrane, 44340, Bouguanias \\ ${ }^{7}$ Naval Energies, 180 rue de Kerlaurent 29490 Guipavas \\ ${ }^{8}$ Dynamocean, 57 rue de Metz, 56000 Vannes \\ ${ }^{9}$ Innosea, 1 rue de la Noë CS 1210244321 Nantes CEDEX 03 \\ ${ }^{10}$ LOMC, UMR 6294, 53, Rue Prony, BP540 - F76058 Le Havre
}

\begin{abstract}
Tidal turbine will be installed in area with high current and high turbulence level. A characterisation of this last is required. The aim of the project THYMOTE is to characterize and understand the generation of eddies from smaller to several tens of meters. Three technics are used: Numerical modelling, Physical modelling, field measurements. Physical and numerical modelling show clearly the appearance of the eddies close to the bottom in presence of dunes or rocks and their motion towards the free surface.
\end{abstract}

\section{Introduction}

Tidal turbines will be deployed at sites subjected to strong currents. This leads to an increased interest in such energetic marine areas. The turbulence is generally high in these areas and the appearance of large eddies was observed at the free surface. The effect of eddies on the turbines should not be the same following its length scale [1].

Turbulence and larges eddies are also appearing in rivers [2] due to the presence of submerged dunes. Both the bed irregularities and their size impact the flow characteristics. Moreover, when the ratio of roughness' to the depth is of the order of $0.3[3,4]$ large-scale vortices are generated that can be moved toward the free surface and are affecting the flow

\footnotetext{
*Corresponding author: sylvain.guillou@unicaen.fr
} 
all along the water column. The review of Best [5] described the state of the knowledge of the dynamics of river dunes.

In tidal energy sites there is no sand dune. In a part of the Raz Blanchard (Fig. 1), the seabed is constituted of rocks and rock outcrops whereas in another part it is constituted of pebbles and rock. The ratio of irregularities to the depth is from 0.02 to 0.15 . The effects of this kind of bottom is not well understood. It is admitted that the conjunction of a complex morphology and strong currents favours a high level of turbulence. A better understanding of the turbulence in powerful sites is therefore essential for tidal energy projects.

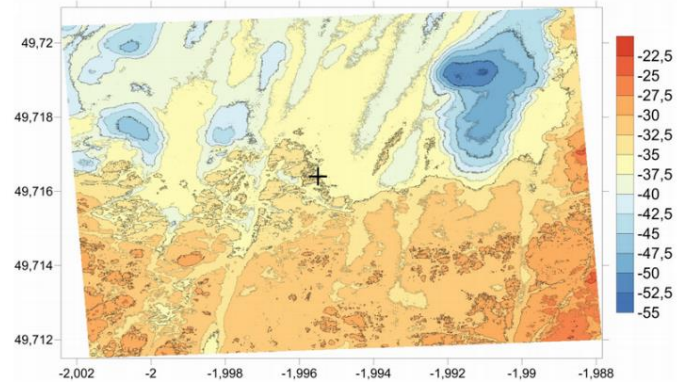

Fig. 1. Bottom morphology in the area of the pilot tidal turbine farm in the Raz Blanchard

\section{Material and Methods}

The aim of the ANR ITE/FEM THYMOTE (2016-2019) project is to study the effect of the bottom morphology on the turbulence in the water column and their potential impacts on the tidal turbines that could be put there. The study site is the Raz Blanchard which represents the highest resource in France. It is located in the English Channel between La Hague Cap and the Alderney island. The main approaches used are (Fig2): in-site measurements [6], flume experiments [7] and numerical simulations [8,9].

a)

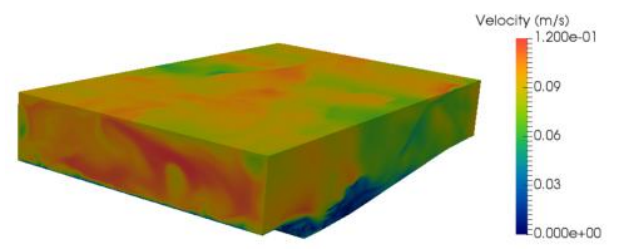

d)

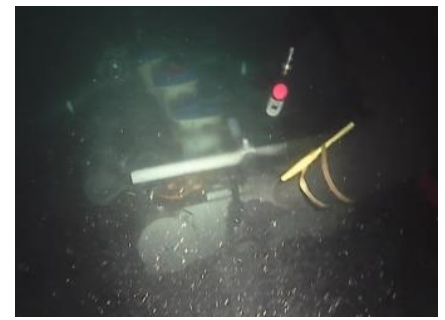

b)

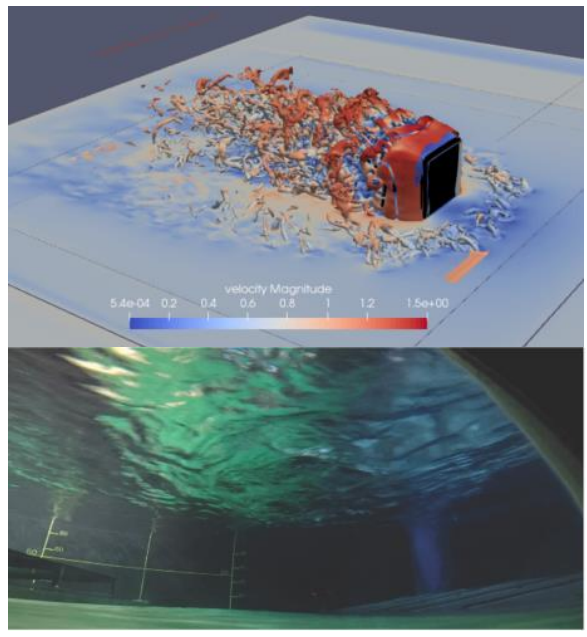

Fig. 2. a) Velocity above a dune obtained with TELEMAC-LES, b) apparition of eddies behind a cube obtained with LBM-LES model, c) large vortex generated by the bottom reaching the free surface, d) ADVPs during the depose operation in the Raz Blanchard.

Two kinds of Large Eddy Simulation (LES) were developed. The first one allows to simulate the eddies at the scale of the race (regional scale) and could observe the impacts of 
large bottom morphology on the flow (Fig. 2a). The Numerical Environmental model TELEMAC was improved. The second one allows to observe the evolution of eddies generated by local irregularities of the bottom such as faults (Fig. 2b). A Lattice Boltzmann Method was developed. In parallel to this last simulations, flume experiments were conducted in the IFREMER laboratory of Boulogne by Particle Image Velocimetry and Laser Doppler Velocimetry (Fig. 2c). Two Acoustic Current Doppler Profilers (ADCP) were user to measure the flow and the turbulence during autumn 2017 (Fig. 2d).

Both Numerical simulations and flume experimentations indicate the generation of the large eddies and their transition towards the free surface. A characterisation of the flow was done in the flume and reproduce with the LES-LBM model successfully. A better understanding of the generation of the eddies responsible for the Kolk boils is in process. The field measurements of the turbulence are in treatment and should provide a lot of information.

This work is funding by French Research Agency (Agence National de la Recherche) program under a contract with the reference number ANR-10-IEED-0006-11.

\section{References}

1. Clark T.H.E. (2015), Turbulence in Marine Environments (TiME): A framework for understanding turbulence and its effects on tidal devices. Proceedings of the 11th European Wave and Tidal Energy Conference, Nantes, 08B4-3-1.

2. Robert A. and Uhlman W., An experimental study on the ripple-dune transition, Earth. Surf. Proc. Landf. 26 (2001), pp. 615-629.

3. Schindler R.J. and Robert A., Flow and turbulence structure across the ripple-dune transition: An experiment under mobile bed conditions, Sedimentology 52 (2005), pp. $627-649$.

4. Omidyeganeh M. and Piomelli U., Large-eddy simulation of two-dimensional dunes in a steady, unidirectional flow, Journal of Turbulence, vol. 12 (42), 2011, 1-31.

5. Best J., The fluid dynamics of river dunes: A review and some future research, directions. Journal of Geophysical Research: Earth Surface, 110(F4), 2005.

6. Pieterse A., Filipot JF, Maisondieu C., Kilcher L., Chaplain N., Coupled ADCP Measurements for Tidal Turbulence Characterization, 12th European Wave and Tidal Energy Conference Proceeding, Cork, 28-31 August 2017, \#894, 8p.

7. Ikheunnicheu M., Germain G., Gaurier B., Druault P., Experimental Tidal Power Site Bathymetry Representation for Turbulence Characterization, 12th European Wave and Tidal Energy Conference Proceeding, Cork, 28-31 August 2017, \#771, 8p.

8. Mercier P., Grondeau M., Guillou S.S., Thiébot J., Poizot E., Toward the Modelling of Turbulence at Tidal Stream Power Sites with the Lattice Boltzmann Method,12th European Wave and Tidal Energy Conference Proceeding, Cork, 28-31 August 2017, \#892, 8p.

9. Bourgoin A., Guillou S.S., Ata R., Thiébot J., Benhamadouche S., Development of a Large-Eddy Simulation Approach for Tidal Currents Modelling, 12th European Wave and Tidal Energy Conference Proceeding, Cork, 28-31 August 2017, \#711, 8p.

10. Milne IA, Sharma RN, Flay RGJ, Bickerton S. Characteristics of the turbulence in the flow at a tidal stream power site. Philosophical transactions of the royal society A: mathematical. Phys Eng Sci 2013; 371:1-14. 